\title{
Transient Fokker-Planck-Kolmogorov equation solved with smoothed particle hydrodynamics method
}

\author{
T. Canor ${ }^{\mathrm{a}, \mathrm{b}, *}$, V. Denoël ${ }^{\mathrm{a}}$ \\ ${ }^{a}$ University of Liège, Department of Architecture, Geology, Environment and Constructions, Structural Engineering \\ Division, Chemin des Chevreuils, 1, B52/3, 4000 Liège, Belgium \\ ${ }^{b}$ F.R.S.-FNRS, National Fund for Scientific Research, 1000 Bruxelles, Belgium
}

\begin{abstract}
Probabilistic theories aim at describing the properties of systems subjected to random excitations by means of statistical characteristics such as the probability density function $\psi$ (pdf). The time evolution of the pdf of the response of a randomly excited deterministic system is commonly described with the transient Fokker-Planck-Kolmogorov equation (FPK). The FPK equation is a conservation equation of a hypothetical or abstract fluid, which models the transport of probability. This paper presents a generalized formalism for the resolution of the transient FPK equation using the well-known mesh-free Lagrangian method, Smoothed Particle Hydrodynamics (SPH).

Numerical implementation shows notable advantages of this method in an unbounded state space: (i) the conservation of total probability in the state space is explicitly written, (ii) no artifact is required to manage far-field boundary conditions, (iii) the positivity of the pdf is ensured and (iv) the extension to higher dimensions is straightforward.

Furthermore, thanks to the moving particles, this method is adapted for a large kind of initial conditions, even slightly dispersed distributions. The FPK equation is solved without any a priori knowledge of the stationary distribution; just a precise representation of the initial distribution is required.
\end{abstract}

Keywords: Fokker-Planck equation, Smoothed Particle Hydrodynamics (SPH) method, Random Dynamics, Meshless Method, Stochastic Process 2008 MSC: xxx.xxx

\section{Introduction}

\subsection{Diffusive processes and FPK equation}

In many engineering matters, systems are subjected to random excitations. Probabilistic theories aim at describing the time evolution of the random properties of a system by means of statistical characteristics such as probability density functions $\psi$ (pdf) or cumulants [1]. For a deterministic system driven by random loadings, governed by diffusive equations, the time evolution of the pdf of its response is given by the transient Fokker-Planck-Kolmogorov (FPK) equation.

This paper is concerned with deterministic systems excited by random inputs. The time evolution of the random state vector $\mathbf{X}(t) \in \mathbb{R}^{n}$ is mathematically described by a stochastic differential equation $[2]$

$$
d \mathbf{X}=\mathbf{f} d t+\mathbf{g} d \mathbf{B}
$$

\footnotetext{
${ }^{*}$ Corresponding author

Email addresses: t.canor@ulg.ac.be (T. Canor), v.denoel@ulg.ac.be (V. Denoël)
} 
where $\mathbf{f}(t, \mathbf{X}(t)): \mathbb{R} \times \mathbb{R}^{n} \rightarrow \mathbb{R}^{n}$ is a nonlinear vector function, $\mathbf{g}(t, \mathbf{X}(t)): \mathbb{R} \times \mathbb{R}^{n} \rightarrow \mathbb{R}^{n \times m}$ a nonlinear matrix function and $\mathbf{W}(t)=d \mathbf{B} / d t$ an $m$-dimensional vector of independent delta-correlated processes. The FPK equation expresses the diffusion of probability density of $\mathbf{X}(t)$ and the conservation of the total probability [3]. It takes the form of a second order partial differential equation

$$
\frac{\partial \psi}{\partial t}=-\sum_{i=1}^{n} \frac{\partial}{\partial x_{i}}\left(\tilde{f}_{i} \psi\right)+\sum_{i=1}^{n} \sum_{j=1}^{n} \frac{\partial^{2}}{\partial x_{i} \partial x_{j}}\left(D_{i j} \psi\right)
$$

where $\psi(t, \mathbf{x}): \mathbb{R} \times \mathbb{R}^{n} \rightarrow[0,+\infty]$ is the joint-pdf of components of $\mathbf{X}(t)$, with initial state distribution $\psi_{0}(\mathbf{x})$, respecting the first two axioms of Kolmogorov

$$
\int_{\mathbb{R}^{n}} \psi d \mathbf{x}=1 \quad \text { and } \quad \psi(\mathbf{x}, t)>0, \forall \mathbf{x} \in \mathbb{R}^{n}
$$

and where $\tilde{f}_{i}(t, \mathbf{x})$ are the drift coefficients (according to Stratonovitch's definition [4]) and $D_{i j}(t, \mathbf{x})$ the diffusion coefficients defined as $2 D_{i j}=\left(\mathbf{g g}^{T}\right)_{i j}$. For a dynamical system, the FPK equation models the transport of the probability density of the response across the state space of the system. The distinction between $\tilde{f}_{i}(t, \mathbf{x})$ and $f_{i}(t, \mathbf{x})$, due to the conceptual definition of the stochastic differential equations, is noteworthy in the event of some systems excited by multiplicative random excitations [5]. A well-posed problem is obtained by complementing Equation (2) with the far-field conditions $\lim _{\|\mathbf{x}\| \rightarrow+\infty} \psi \tilde{f}_{i}=0, \lim _{\|\mathbf{x}\| \rightarrow+\infty} \psi \frac{\partial}{\partial x_{i}} D_{i j}=0$ and $\lim _{\|\mathbf{x}\| \rightarrow+\infty} \psi D_{i j}=0$ at any time $t[3]$.

\subsection{Solution of FPK equation}

The common method to solve stochastic differential equations consists in simulating samples of the response of the system [6]. Then, moments and cumulants are computed with statistical postprocessing. However, the rate of convergence decreases with the desired statistical order [7]. For years, many semi-analytical methods have been developed to avoid plethora of simulations, as cumulantneglect closures [1], statistical linearization [8,9] or stochastic averaging [1, 7], but they turn out to be too approximate or unstable in some applications. From this viewpoint, the resolution of FPK equation remains attractive, because a pdf contains all the statistical characteristics of a random vector and gives some information about the tail distribution.

The exact explicit solution for transient FPK equation may be obtained in only few cases, in particular for linear systems driven by Gaussian excitation and some nonlinear Hamiltonian systems [3]. Nevertheless, for most of the nonlinear systems, such exact solutions cannot be calculated explicitly and the use of numerical methods is inevitable [10].

The first numerical technique used to solve FPK equation was the finite difference method [11, 12]. In further attempts, the finite element (FE) method has also been used [13, 14]; it has been extended up to a four-dimensional state space [15]. This method allows to solve the unsteady equation through a step-by-step analysis or immediately the stationary equation through an eigenvalue problem. Nevertheless, the finite element method encounters difficulties in modeling the far-field boundary conditions. To overcome this problem, Spencer [13] proposes to mesh a sufficiently large domain to eliminate the diffusion of probability. Langtangen [16] proposes to impose a zero flux condition at the boundaries of the integration domain. In any case, the positivity of the pdf is not properly ensured: if elements are too large or the domain too small some spurious waves can propagate through the state space and spoil the quality of the solution. This FE method may thus fail in creating artificially and numerically the vanishing condition $\psi(t, \mathbf{x}) \rightarrow 0$ for $\|\mathbf{x}\| \rightarrow+\infty$ at any time $t$. Furthermore, the finite element method is extended with difficulties to high multidimensional systems, because of some difficulties to discretize high-dimensional state spaces. Another argument out of favor with mesh-based methods is the need to mesh useless subspaces: at a certain time step, probability density in some subspaces is so low that its representation is useless. For a given computing capacity, it is more useful to refine appropriate zones of the state space. 
To circumvent the issues encountered with mesh-based methods, recent developments propose to apply meshless methods to the resolution of FPK equation. Kumar et al. [17] propose the use a multiresolution meshless method based on Meshless Petrov-Galerkin method, but this method requires an important time of execution and the convergence is an issue, as pointed in [18]. Then, Kumar et al. propose the use of PUFEM (partition of unity finite element method) and apply it to the stationary [18] and the transient [19] FKP equation up to a four-dimensional state space. The examples illustrating the method are classical nonlinear mechanical systems as Duffing oscillator or Lorenz attractor.

In the field of kinetic theory, the FPK equation presents a great interest for modeling complex fluid dynamics. Chauvière and Lozinski [20, 21] present a spectral discretization approach to solve FPK equation, another mesh-based method. In this approach, some drawbacks similar to the FE method may be encountered as the dependence of the positivity with the refinement of the mesh and instability for high gradient in the pdf [21]. With the objective of solving multidimensional partial differential equations, Ammar et al. [22, 23, 24] develop a method based on a separate representation of variables, which allows to decouple the numerical integration in each dimension of the system. This method shows encouraging results for high-dimensional time-invariant systems on a bounded domain. In complex fluid theory, the definition of FPK equation differs from Equation (1) in the domain of the state vector $\mathbf{X}(t)$ which is bounded [25]. The occurrence of rare events is not a major issue in this field as it is the case in mechanical or civil engineering [1] and so the question of the tail distribution is not addressed. Furthermore, in a bounded domain, the stability and the efficiency of the method is not the same issue as it is in an unbounded state space.

The time dependence of systems modeled with Equation (1) is explicitly considered in the time dependence of drift and diffusion coefficients. Some of these methods may also reveal some numerical drawbacks in the resolution of FPK equation related to time-variant systems. In the FE method or in the PUFEM approach, the matrices of the system must be computed at each time step and the modal reduction for transient problem is not possible anymore. And yet, the explicit time dependence of drift and diffusion coefficients is a basic problem in transient dynamics. For example, in earthquake engineering [26], the seismic excitation is usually modeled as a diffusive process modulated by a time window. Therefore, the FPK equation has explicit time-dependent coefficients.

\subsection{Aims of this work}

In this work, a particle strategy is explored to deal with the FPK equation related to dynamical diffusive systems. The FPK equation is viewed as a convection-diffusion equation in a Lagrangian paradigm. The smoothed particle hydrodynamics (SPH) method, a meshfree particle method, is used for the resolution of FPK equation.

An efficient method for solving the transient FPK equation in an unbounded domain must be able (i) to adaptively cover all the state space from initial condition to steady-state, (ii) to ensure the vanishing condition in the far field (iii) to precisely capture important distortions of the pdf and (iv) to assuredly maintain the positivity of pdf. The SPH method, with the implementation details given next, fulfills all these requirements.

The SPH method, as other particle methods, has been widely used for the resolution of diffusion equations. This method was developed by Lucy in 1977 [27] (and simultaneously by Gringold and Monaghan [28]) and first applied to astrophysical problems. Thus far, the method has been applied to many problems of continuous and discontinuous dynamics as fluid flow problems [29, 30], damage and fracture [31, 32], impact computation [33] or heat conduction [34, 35]. The main principles of the SPH method are widely developed in [36, 37, 38, 39]. In the context of kinetic theory, Chaubal et al. [40, 41] applies first SPH technique to the dynamics of liquid crystalline polymers on a closed domain. Particle strategies for solving the Fokker-Planck equation are commonly used in this theory, as illustrated in $[22,42,43]$. Inspired by these previous works, the present paper proposes an application of the SPH formalism to solve the transient FPK equation related to diffusive random systems independently of their size. In this context, a new formulation of the conservation equation using the Lagrangian formalism is proposed and some considerations to deal with the vanishing condition are exposed. 
In the following sections, the SPH method is briefly exposed, then the FPK equation is transformed into the SPH formalism. Finally, the developments are applied to dynamical systems usually encountered in the literature, but also to more original problems, and the main results are discussed.

\section{Smoothed particle hydrodynamics}

The philosophy of the SPH method is to transform a set of partial differential equations into integral equations by using an interpolation function that gives the kernel estimation of a field (density, velocity, energy) at a point. Vector or scalar fields are only known in a number of discrete points. Thus, the evaluation of an integral is transformed into a sum over some neighboring particles. In this method, a grid is unnecessary. Indeed, interactions between neighboring particles are modeled with interpolation functions, so-called kernel functions, which depend on the distance between particles.

\subsection{Integral representation of a field}

The concept of integral representation of a continuous field $f(\mathbf{x})$ in SPH method uses the properties of the Dirac-delta function $\delta(\mathbf{x})$,

$$
\int_{\mathbb{R}^{n}} f\left(\mathbf{x}^{\prime}\right) \delta\left(\mathbf{x}-\mathbf{x}^{\prime}\right) d \mathbf{x}^{\prime}=f(\mathbf{x})
$$

with $\delta\left(\mathbf{x}-\mathbf{x}^{\prime}\right)=0$ if $\mathbf{x} \neq \mathbf{x}^{\prime}$. This function cannot be numerically represented. Therefore, the Dirac-delta function is replaced by the kernel function $W\left(\left|\mathbf{x}-\mathbf{x}^{\prime}\right|, h\right)$, such that

$$
\lim _{h \rightarrow 0} W\left(\left|\mathbf{x}-\mathbf{x}^{\prime}\right|, h\right)=\delta\left(\mathbf{x}-\mathbf{x}^{\prime}\right)
$$

with $h$ the smoothing length. The kernel approximation of $f(\mathbf{x})$ with the kernel function is

$$
<f(\mathbf{x})>=\int_{\mathbb{R}^{n}} f\left(\mathbf{x}^{\prime}\right) W\left(\left|\mathbf{x}-\mathbf{x}^{\prime}\right|, h\right) d \mathbf{x}^{\prime} .
$$

According to the Dirac-delta function property $(5),<f(\mathbf{x})>$ tends to $f(\mathbf{x})$ when $h$ tends to zero. This latter expression is a smoothed approximation of $f(\mathbf{x})$ and the integral is discretized, for a number $N_{p}$ of particles, as

$$
<f(\mathbf{x})>\approx \sum_{j=1}^{N_{p}} f\left(\mathbf{x}_{j}\right) W\left(\left|\mathbf{x}-\mathbf{x}_{j}\right|, h\right) \Delta V_{j}=\sum_{j=1}^{N_{p}} \frac{m_{j}}{\rho_{j}} f\left(\mathbf{x}_{j}\right) W\left(\left|\mathbf{x}-\mathbf{x}_{j}\right|, h\right)
$$

where $m_{j}, \rho_{j}$ and $\Delta V_{j}$ are the mass, the density and the volume of particle $j$, respectively. Equation (7) is the particle approximation of the field $f(\mathbf{x})$. This approximation can be particularized at a particle $i$,

$$
<f\left(\mathbf{x}_{i}\right)>=\sum_{j=1}^{N_{p}} f\left(\mathbf{x}_{j}\right) W_{i j} \Delta V_{j}
$$

with $W_{i j}=W\left(r_{i j}, h_{i}\right)$ and $r_{i j}$ the distance between particles $i$ and $j$. Equation (8) highlights the difference between the particle value $f\left(\mathbf{x}_{i}\right)$ and the kernel approximation $\left\langle f\left(\mathbf{x}_{i}\right)\right\rangle$. These values must not be confused [29], but they can be sufficiently close if $h$ is small enough according to (5) and $(6)$. 


\subsection{Kernel function}

To be used in SPH method, a kernel function must fulfill some conditions [44]:

- Unity: the kernel function must be normalized

$$
\int_{\mathbb{R}^{n}} W\left(\left|\mathbf{x}-\mathbf{x}^{\prime}\right|, h\right) d \mathbf{x}^{\prime}=1 \quad \text { or } \quad \sum_{j=1}^{N} W\left(\left|\mathbf{x}-\mathbf{x}_{j}\right|, h\right) \Delta V_{j}=1
$$

- Delta function property as formulated in (5),

- Compact support: $W(r, h)=0$ for $r \geq \kappa h$, where $\kappa$ is a constant depending on the kernel function,

- Decay: the smoothing function should decrease monotonically with the distance between a particle and the neighboring ones,

- Symmetry, positivity, smoothness.

In this work, the original Lucy kernel function is used. Introduced by Lucy in 1977 [27], this kernel is a polynomial function continuously differentiable over its compact support $(\kappa=1)$,

$$
W(R, h)=\alpha_{d}\left\{\begin{array}{cc}
(1+3 R)(1-R)^{3}, & \text { if } R \leq 1 \\
0, & \text { if } R>1
\end{array}\right.
$$

with $R=r / h$. The parameter $\alpha_{d}$ depends on the dimension $n$ of the state space. From (9), $\alpha_{d}$ is $5 / \pi h^{2}, 105 / 16 \pi h^{3}$ and $28 / \pi^{2} h^{4}$ in a two-, three- or four-dimensional space, respectively.

\section{SPH formulation of Fokker-Planck-Kolmogorov equation}

\subsection{Lagrangian formalism}

In the context of fluid dynamics, an Eulerian description of motion can be imagined as that given by a fixed observer looking at the evolution of fluid properties in a finite, fixed and undeformable volume. Contrarily, a Lagrangian description of motion can be viewed as the motion described by observers sitting on moving particles. These views modify the formulation and the interpretation of the equations of motion: in a Lagrangian formalism, the integration points are moving according to trajectories depending on the modeled system.

The Eulerian formulation of a convection-diffusion phenomenon of a scalar field $\phi$ is transformed into a Lagrangian formulation by introducing the concept of material derivative $D / D t$,

$$
\frac{D \phi}{D t}=\frac{\partial \phi}{\partial t}+\mathbf{v} \cdot(\nabla \phi)
$$

where $\mathbf{v}$ is the medium velocity.

The FPK equation (2), as a convection-diffusion equation, can be recast into such a Lagrangian formalism. Indeed, Equation (2) can be written as

$$
\frac{\partial \psi}{\partial t}=-\sum_{i=1}^{n} \frac{\partial}{\partial x_{i}}\left(\psi\left(\tilde{f}_{i}-\sum_{j=1}^{n} \frac{\partial D_{i j}}{\partial x_{j}}-\frac{1}{\psi} \sum_{j=1}^{n} D_{i j} \frac{\partial \psi}{\partial x_{j}}\right)\right)=-\sum_{i=1}^{n} \frac{\partial}{\partial x_{i}}\left(v_{i} \psi\right)
$$

where it is readily seen that the $i$-th component of $\mathbf{v}(\mathbf{X}, t)$ defined as

$$
v_{i}=\tilde{f}_{i}-\sum_{j=1}^{n} \frac{\partial D_{i j}}{\partial x_{j}}-\frac{1}{\psi} \sum_{j=1}^{n} D_{i j} \frac{\partial \psi}{\partial x_{j}} .
$$

provides a form similar to (11). The diffusion velocity $\mathbf{v}[45,46]$ has a term divided by $\psi$, but there is no hidden difficulties. Indeed, if $\psi$ represents a density of probability, i.e. a quantity of probability 
divided by a given volume, a particle cannot have a density equal to zero because it cannot have a mass equal to zero (as shown in section 3.3); nor can it occupy an infinite volume.

The conservation equation (12) is further developed to introduce the total derivative

$$
\frac{\partial \psi}{\partial t}+\sum_{i=1}^{n} \frac{\partial}{\partial x_{i}}\left(v_{i} \psi\right)=\frac{D \psi}{D t}+\sum_{i=1}^{n} \psi \frac{\partial v_{i}}{\partial x_{i}}=0 .
$$

and the vectorial equation of transport across the state space simply reads

$$
\frac{d \mathbf{X}}{d t}=\mathbf{v}
$$

with $\mathbf{v}$ the velocity field defined in (13).

In this method, the steady-state regime must be interpreted from a Lagrangian perspective: the particle velocity is not null, but the particles keep on moving along $(n-1)$-dimensional isoprobability manifolds. This is shown as follows. In steady-state regime, the material derivative of $\psi$ and the partial derivative with respect to time of $\psi$ are equal to zero. Therefore, Equation (11) leads to $\mathbf{v} \cdot(\nabla \psi)=0$, which shows that the steady-state velocity field is always normal to the gradient of the pdf, like the tangent vector to isoprobability manifolds.

\subsection{Conservation equation}

The quantity of probability $\mu_{j}$ (equivalent to mass in hydrodynamics) carried by a particle $j$ is defined as the product of the particle volume $\Delta V_{j}$ and the particle density $\psi_{j}$. By analogy with fluid dynamics, this quantity of probability is referred to as mass in the following. Taking into account (8), the particle approximation of the density of probability $\psi$ at a particle $i$ is

$$
<\psi\left(\mathbf{x}_{i}\right)>=\sum_{j=1}^{N_{p}} \psi_{j} W_{i j} \Delta V_{j}=\sum_{j=1}^{N_{p}} \mu_{j} W_{i j}
$$

where $\mu_{j}=\psi_{j} \Delta V_{j}$. This particle approximation of the probability density function is equivalent to what is usually done in hydrodynamics [36]. In dedicated literature, Equation (16) is often mentioned as the summation density approach. In the sequel, as the mass of each particle is invariant, the total unit probability is conserved. From a computational point of view, there is no need to solve the conservation equation as a differential equation. Furthermore, the positivity of the pdf is ensured, because each particle approximation is a sum of positive terms $\left(\mu_{j}, W_{i j}>0\right)$.

\subsection{Initialization of particle masses}

The initialization of masses is a key issue in the method. From the arbitrary initial distribution of particles in the state space, the reconstructed field is calculated where particles are initially located $<\psi\left(\mathbf{x}_{j}\right)>$. Then, the solution of (16) should in principle give the particle masses. Nevertheless, for computational efficiency reasons and also because the system (16) may be ill-conditioned, an alternative approach is followed. The masses $\mu_{j}$ and the initial particle values $\psi_{0, j}$ are related by $\mu_{j}=\psi_{0, j} \Delta V_{0, j}$, where $\Delta V_{0, j}$ is the initial volume associated with the $j^{\text {th }}$ particle, which corresponds to a hypercubic subspace if the initial arrangement of particles is regular. Although conceptually different, the initial condition $\psi_{0}$ may be understood as the kernel approximation of the probability density function. Indeed, a reasonable approximation for $\psi_{0, j}$ is the initial reconstructed field $\left\langle\psi_{0}\left(\mathbf{x}_{j}\right)\right\rangle$ provided the smoothing length is small enough according to (6). With this approach, the sum of particle masses is not strictly equal to unity, because of the limited integration domain and the approximation on the integration volumes. However, this error may be reduced by increasing the number of particles and so reducing the smoothing length as presented hereafter. After initialization, the particle masses are kept constant in time. Consequently, with this initialization technique, there is no equation to solve, neither for the initialization of masses, nor during the transient resolution. 


\subsection{Estimation of statistical moments}

The SPH method and the integral representation of pdf also allow an approximation of statistical moments of the random state vector $\mathbf{X}=\left[X_{1} \ldots, X_{n}\right]^{\mathrm{T}}$. Indeed, the particle approximation of statistical moments reads

$$
<E\left[X_{1}^{\alpha_{1}} \ldots X_{n}^{\alpha_{n}}\right]>=\int_{\mathbb{R}^{n}} x_{1}^{\alpha_{1}} \ldots x_{n}^{\alpha_{n}}<\psi(\mathbf{x})>d \mathbf{x}
$$

where $E[\cdot]$ is the expectation operator and $\alpha_{i} \in \mathbb{N}$ for $i=1 \ldots, n$. Therefore, if the masses are reasonably well calculated by the previous relations, Equation (17) in an SPH formalism yields

$$
<E\left[X_{1}^{\alpha_{1}} \ldots X_{n}^{\alpha_{n}}\right]>\approx \sum_{i=1}^{N_{p}}\left(\prod_{k=1}^{n}\left(\mathbf{X}_{i}\right)_{k}^{\alpha_{k}}\right)<\psi_{i}>\Delta V_{i} .
$$

In this approximation technique, particles are seen as integration points. The volume $\Delta V_{i}$ occupied by the $i^{\text {th }}$ particle is also considered as the integration volume. This approximation means that the kernel approximation $\left\langle\psi_{i}\right\rangle$ of the pdf is constant in the volume of the particle. This integration technique is similar to the rectangle integration method. The computation of statistical moments is posterior to the solution of the FPK equation.

The choice of $\Delta V_{i}$ as integration volumes is justified, if the reciprocity in the interaction between particles is imposed. The reciprocity exists, if the matrix $\mathbf{W}$ gathering the elements $W_{i j}$, is symmetric. Actually, the unity principle is expressed as

$$
\Delta V_{j}=\sum_{k=1}^{N_{p}}\left(\mathbf{W}^{-1}\right)_{j k} .
$$

So, if one assumes that the volumes $\Delta V_{j}$ are integration volumes for the kernel approximation $<$ $\psi\left(\mathbf{x}_{j}\right)>$, the conservation of total probability leads to

$$
\sum_{j=1}^{N_{p}}<\psi\left(\mathbf{x}_{j}\right)>\Delta V_{j}=\sum_{i=1}^{N_{p}} \mu_{i}\left(\sum_{j=1}^{N_{p}} \sum_{k=1}^{N_{p}}(\mathbf{W})_{j i}\left(\mathbf{W}^{-1}\right)_{j k}\right) .
$$

The summation (20) is constant if and only if $\mathbf{W}$ is symmetric, which is not ipso facto the case. Among available ways to make $\mathbf{W}$ symmetric, the method adopted here consists in averaging the reciprocal kernel functions between two particles as

$$
W_{i j}=W_{j i}=\frac{1}{2}\left(W\left(r_{i j}, h_{i}\right)+W\left(r_{i j}, h_{j}\right)\right) .
$$

\subsection{Probability of exceedance}

The proposed SPH method also allows the computation of probability function depending on the pdf of the state vector $\mathbf{X}(t)$. The mathematical formulation of a structural reliability problem [26] consists in calculating the probability of exceedance $P_{f}$ defined as the probability integral

$$
P_{f}(t)=\int_{\Omega_{f}} \psi(t, \mathbf{x}) d \mathbf{x}
$$

where $\Omega_{f}$ denotes the subspace where exceedance (or failure) occurs. In the presented SPH method, the calculation of such a probability may be estimated directly: at a given time, only the mass of particles contained in the domain $\Omega_{f}$ contributes to the probability of failure. Therefore, an estimation of the probability of failure $\left\langle P_{f}(t)>\right.$ at a given time $t$ is

$$
<P_{f}(t)>=\sum_{j=1}^{N_{p}} \mu_{j} \mathbb{I}\left(\mathbf{X}_{j}(t) \in \Omega_{f}\right)
$$

where $\mathbb{I}(\mathcal{A})$ is a set function equal to one if the condition $\mathcal{A}$ is fulfilled, and zero otherwise. 


\subsection{Transport equation}

According to (16), the probability density of a particle $i$ depends on $W_{i j}$ and therefore on the relative positions of particles at time $t$. To calculate the position of a particle $i$, Equation (15) is integrated with a first-order forward Euler integration scheme

$$
\mathbf{X}_{i}^{t+\Delta t}=\mathbf{X}_{i}^{t}+\mathbf{v}_{i}^{t} \Delta t
$$

An adaptive time step is implemented to ensure the stability and the accuracy of the method as presented in [47],

$$
\Delta t=C_{r} \min _{i=1, \ldots N_{p}}\left(\frac{\min _{j=1, \ldots N_{p}, j \neq i}\left(r_{i j}\right)}{\left\|\mathbf{v}_{i}\right\|}\right)
$$

with $C_{r}(<1)$ the Courant number. An adaptive time step is necessary in the resolution of FPK equation, at least when particles are initially slightly dispersed, since particles are very close and an inappropriate time step can spoil the distribution of particles or cause collisions between them. This proposed Courant condition may be severe compared with some usual Courant conditions proposed in the literature usually based on the smoothing length and not on the distance between particles [38]. Nevertheless, the Courant criterion as formulated in Equation (25) conserves the particle meaning of this condition. Hence, according to this criterion, a particle in a time step cannot cross a distance greater than a fraction of its distance to the closest neighbor particle. Furthermore, with Equation (25), the time step depends only on geometrical considerations and not on the probability density (as it would be if the smoothing length was considered). From the authors' experience, this option increases the stability performances of the algorithm.

In the numerical implementation, the velocity field is established, according to the concept of integral approximation of a vector field and Equation (13) becomes

$$
\mathbf{v}(\mathbf{X})=\mathbf{f}(\mathbf{X})-\frac{1}{\psi(\mathbf{X})} \mathbf{D} \nabla \psi(\mathbf{X})
$$

so that the particle approximation at time $t$ of Equation (26) is

$$
\mathbf{v}_{i}^{t}=\mathbf{f}\left(\mathbf{X}_{i}^{t}\right)-\frac{1}{<\psi\left(\mathbf{X}_{i}^{t}\right)>} \mathbf{D}<\nabla \psi\left(\mathbf{X}_{i}^{t}\right)>
$$

where $\left\langle\psi\left(\mathbf{X}_{i}^{t}\right)>\right.$ is calculated with (16) and $<\nabla \psi\left(\mathbf{X}_{i}^{t}\right)>$ calculated as

$$
<\nabla \psi\left(\mathbf{X}_{i}^{t}\right)>=\sum_{j=1}^{N_{p}} \mu_{j} \nabla_{i} W_{i j} .
$$

The Lagrangian formalism makes the method relatively robust with respect to the dissemblance between initial and steady-state distributions. With this particle approximation method, particles move from the initial to the stationary distribution by themselves. This is a noticeable difference with mesh-based methods where the mesh must cover a sufficiently large space in order to represent every step of the transient solution.

In SPH method, leapfrog integration scheme is preferred, because it is simply implemented and second-order accurate in both time and space [48]. With the leapfrog algorithm, Equation (15) is integrated as [38]

$$
\mathbf{X}_{i}^{t+\Delta t}=\mathbf{X}_{i}^{t}+\mathbf{v}_{i}^{t+\frac{\Delta t}{2}} \Delta t
$$

with the midpoint velocity field estimated as

$$
\mathbf{v}_{i}^{t+\frac{\Delta t}{2}}=\mathbf{f}\left(\mathbf{X}_{i}^{t+\frac{\Delta t}{2}}\right)-\frac{1}{\left\langle\psi\left(\mathbf{X}_{i}^{t+\frac{\Delta t}{2}}\right)>\right.} \mathbf{D}<\nabla \psi\left(\mathbf{X}_{i}^{t+\frac{\Delta t}{2}}\right)>
$$


and

$$
\mathbf{X}_{i}^{t+\frac{\Delta t}{2}}=\mathbf{X}_{i}^{t}+\mathbf{v}_{i}^{t} \frac{\Delta t}{2}
$$

The choice between an Euler explicit scheme and a leapfrog scheme is typically driven by accuracy and computation efficiency. On the one hand, if accuracy is leading, the leapfrog algorithm is more efficient for the same adaptive time step procedure. On the other hand, if the choice is motivated by efficiency, Euler's scheme is more advantageous, because the estimation of the velocity field, i.e. the more consuming operation, is performed just once per time step. In any case, the selection of a small time step reduces the error due to the time discretization but barely influences the residual error due to particle interpolation, as shown in the following illustrations.

\subsection{Particle interaction and smoothing length}

The determination of the smoothing length $h$ is an important issue in the SPH method. If the smoothing length is too small, a particle does not interact with its neighboring particles. On the other hand, if the smoothing length is too large, local properties of the studied field could be excessively smoothed. It is commonly assumed that the accuracy depends on $h^{2}$ [38].

The smoothing length also influences the computation time. For these reasons, the smoothing length associated to each particle is adapted at each time step. Liu [38] suggests to maintain an appropriate number of particles in the neighborhood of each particle: 5, 21 and 57 in one-, two- and three-dimensions, respectively.

To adapt the smoothing length with particle positions, different authors suggest relations to maintain the number of neighboring particles constant [38]. The simplest relation consists in keeping the initial product $\left(h_{0} \sqrt[n]{\psi_{0}}\right)_{i}$ constant in time for each particle, where $n$ is the dimension of the considered space. The smoothing length is updated with the evolution of $\psi_{i}$, as

$$
h_{i}=h_{0, i}\left(\sqrt[n]{\frac{\psi_{0}}{\psi}}\right)_{i} .
$$

Equation (32) expresses also the limited risk that a particle gets isolated. Indeed, if particles are too close, their densities increase and their smoothing lengths decrease. Conversely, if a particle is too distant from the others, its density decreases and its smoothing length increases. Therefore, a particle cannot be isolated without any interaction with its neighbors.

Smoothing length and particle interaction are closely related in SPH method. Indeed, a particle $i$ interacts only with particles contained in its compact support. Therefore, it is useless to check possible interaction if two particles are too far from each other. Specific literature widely covers this topic $[37,38]$.

The discussion about particle interaction and smoothing length only makes sense in the standard version of the method based on Eulerian kernels. In this approach, the compact support has a constant shape (but the size is updated) in time, but the particles present in this support change. Besides, the particle interaction may also be interpreted in a Lagrangian paradigm. A major difficulty in SPH method with Eulerian kernel concerns the tensile instability [49] which occurs in elasticity problems when particles tend to clump in pairs under tension. This instability is mathematically due to the calculation of the second derivative of the kernel [50]. A fully Lagrangian kernel [51] or an updated Lagrangian kernel [52] can be used to discard occurence of this instability. The Lagragian kernel is a robust and stable method to deal with solid mechanics. Nevertheless, this method is not suitable for fluid flow problems [51], because the deformation can be much larger in fluids than in solids. In our particular case, the transient FPK equation, as a convection-diffusion equation, is closer to the modeling of an artificial fluid without viscosity or constitutive law than an elastic solid, a reason why we have opted for Eulerian kernels. In spite of some drawbacks, an Eulerian kernel also offers a simple management of the mixing of particles or the large distortions as illustrated hereafter. 


\subsection{Boundary conditions}

In the standard SPH method, the treatment of boundary conditions is sometimes neglected, as in astrophysical problems [27] which deal with theoretically infinite domains. In the treatment of free surface boundary conditions, the standard SPH method behaves quite well, because the deficiency of particles near the boundary acts as a zero pressure condition. Nevertheless, the term "free surface" is not suitable for this application, because there is no clear separation between two media as in fluid or solid and the probability density function is defined everywhere in the state space.

In the normalized SPH method, Randles and Libersky [36] suggest to define the density of a boundary particle with the kernel summation corrected with regard to the boundary deficiency. In this method, the definition of boundary particles remains an issue, especially for large modification of particle distribution compared with the initial one. Furthermore, the estimation of the gradient is not relevant.

In our context with only far-field conditions, it is proposed to define an initial continuous probability distribution on a sufficiently large domain to have surrounding particles with a very low mass. These peripheral particles introduce implicitly a condition similar to $\psi(t, \mathbf{x}) \rightarrow 0$ when $\|\mathbf{x}\| \rightarrow \infty$. Their interactions with inner particles of higher masses are limited. So, the absence of boundary treatment in the far field may be justified in this manner.

\subsection{Short description of the algorithm}

Initially the particle are regularly spread out and the initial pdf is calculated at the position of each particle. A regular initial distribution allows to choose a smoothing length $h$ for each particle that ensures an adequate number of neighboring particles in the compact support. Masses $\mu_{j}$ are initialized as in section 3.3 and are kept constant throughout the resolution.

In a time loop, the computation of the conservation equation (16) and the gradient of the pdf (28) requires to find as fast as possible the particles contained in the compact support of a given particle. This operation becomes time consuming if the number of particles is too important. The implemented algorithm, presented hereafter, is based on a cell mapping [38], which is commonly used in SPH applications.

To compute interactions, particles are first sorted in cells. This mapping consists in dividing the state space in a number of cells or $n$-dimensional hypercubes. In some applications, the edge-size of hypercubes is chosen as the maximum smoothing length $h$ over all particles. This choice is adequate only if the density in the system is rather homogeneous. In the case of probability density evolution, there are large differences between zones of the state space. Therefore, the mapping is constructed with a given number of cells and the size of edges, identical for each cell, are modified at each time step. The method consists in exploiting the regularity of the cell map to find the smaller set of cells including completely the hypercube centered on the particle and with an edge equal to the diameter of the compact support.

Finally, when the kernel approximations of the fields $\psi(\mathbf{x})$ and $\nabla \psi(\mathbf{x})$ are calculated at each particle position, the velocity field (27) is evaluated for each particle and the particle positions are immediately modified according to (15). At this step, the smoothing length and the time step are updated according to Equations (32) and (25), respectively.

\section{Illustrations}

\subsection{Validation on a SDOF linear oscillator}

First, to illustrate and validate the method, a dimensionless SDOF linear oscillator is considered,

$$
\ddot{x}+2 \xi \dot{x}+x=W
$$

where $W(t)$ is a normalized Gaussian $\delta$-correlated process with zero mean and $E[W(t) W(t+\tau)]=\delta(\tau)$ and $\xi$ is the damping ratio equal to 0.1 in this example. The exact solution of this problem, aiming 
at determining the time evolution of first and second-order moments is obtained for instance with Gaussian closure method [1]. The initial uncorrelated Gaussian distribution has mean position and mean velocity equal to 1 and standard deviations of position and velocity equal to 0.1 , in order to demonstrate that the developed method is able to cope with initial conditions with few dispersion.

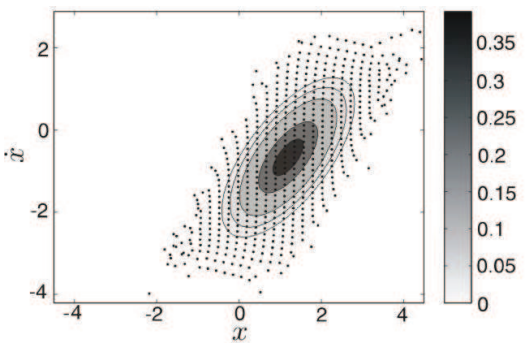

(a)

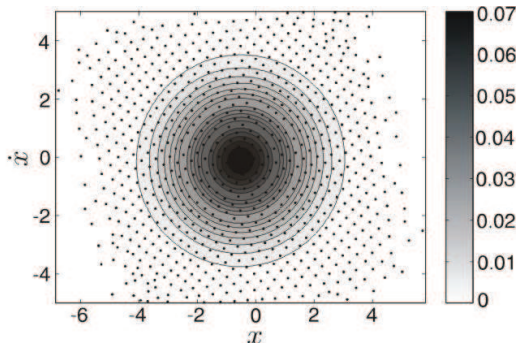

(b)

Figure 1: Reconstructed probability density function $\psi$ and position of particles at (a) $t=1.3$ and (b) $t=10$ for $N_{p}=1681$.

Figure 1 presents two snapshots at different times of the pdf reconstructed from the particle positions. The particles automatically move from the initially meshed zone to the subspace of interest at any time step and they finally reach the steady-state distribution.

In order to study the accuracy of the method, the FPK equation is solved for different numbers of particles $N_{p}$ and a Courant number $C_{r}$ fixed to 0.2 . The relative error on the pdf is calculated for each time step as

$$
\mathcal{E}_{\psi}=\frac{\left\|<\psi>-\psi_{\text {ana }}\right\|_{2}}{\left\|\psi_{\text {ana }}\right\|_{2}}=\frac{\sqrt{\sum_{j=1}^{N_{p}}\left|<\psi>-\psi_{\text {ana }}\right|_{j}^{2}}}{\sqrt{\sum_{j=1}^{N_{p}}\left|\psi_{\text {ana }}\right|_{j}^{2}}}
$$

with $\psi_{\text {ana }}(t, \mathbf{x}(t)): \mathbb{R} \times \mathbb{R}^{2} \rightarrow \mathbb{R}_{0}^{+}$the analytical solution of the FPK equation related to (33). In Figure 2-a the time evolution of the error $\mathcal{E}_{\psi}$ for three numbers of particles $N_{p}$ is shown. For a small number of particles, the error $\mathcal{E}_{\psi}$ is rather significant in the early transient phase, but it decreases when $N_{p}$ increases, because the smoothing length decreases.

The second-order moment of $x$ calculated with Equation (18) is illustrated in Figure 2-b. In the early transient phase, it is well estimated, even with a small number of particles. In the steady-state distribution, there are less particles in the bulk of the pdf and a larger amount of particles is necessary for an accurate estimation of the second moment. Nevertheless, instead of using (18), translating the rectangle approximation, a better approach consists in interpolating pdf values between the values calculated at particle positions and then to calculate moments with (17). This operation reduces the difference between the exact moment and the approximation (calculated with $N_{p}$ equal to 1681), as illustrated in Figure 2-b. 


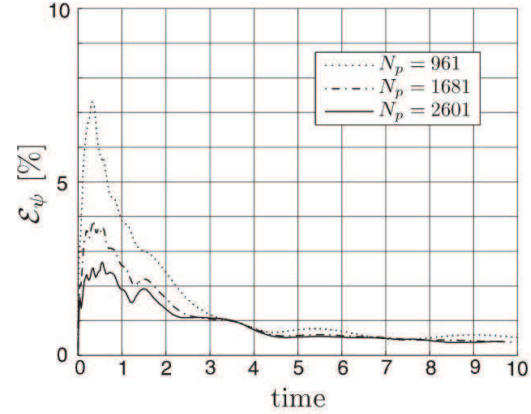

(a)

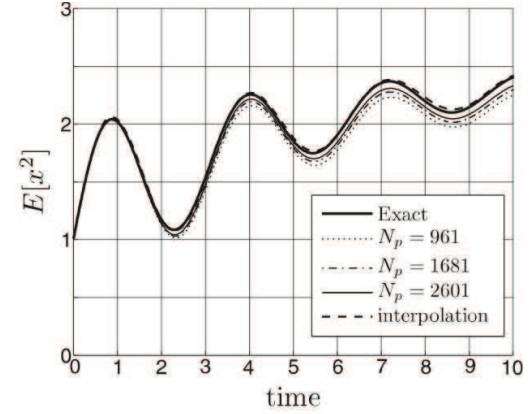

(b)

Figure 2: Two-dimensional linear system: (a) relative error on the pdf $\mathcal{E}_{\psi}$ with respect to time as defined in (34), (b) the time evolution of the second-order moment of $x$, noted $E\left[x^{2}\right]$.

The reduction of the errors while increasing $N_{p}$ implies also an increase of the computation time for two different reasons: (i) the computation of the particle interaction increases and (ii) the particles are closer so the time step is reduced with regard to Equation (25).

Figure 3 shows the influence of time integration parameters on the error $\mathcal{E}_{\psi}$ : the influence of the Courant number and the integration scheme are illustrated in this example. Figure 3-a compares the error $\mathcal{E}_{\psi}$ committed with an Euler explicit scheme and different Courant numbers. The error is not significantly influenced by this parameter, even if the error is less for Courant numbers lower than 0.5. Figure 3-b compares the two integration schemes previously presented. For the comparison, the time step is chosen constant and small enough to keep the Courant number lower than 1 for the whole computation. As announced previously, the reduction of the error between an Euler explicit integration scheme and a leapfrog algorithm is not significant. The residual error on $\psi$ preponderantly depends on the particle discretization and not on the time integration.

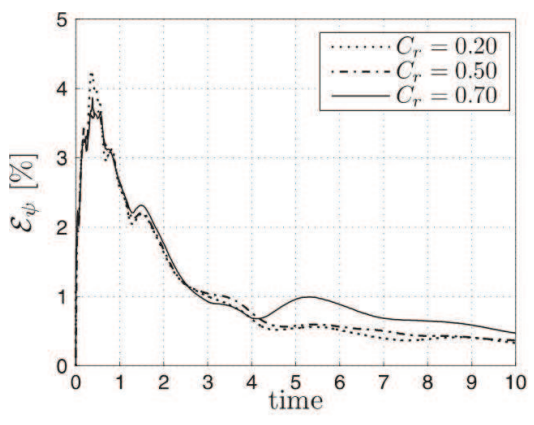

(a)

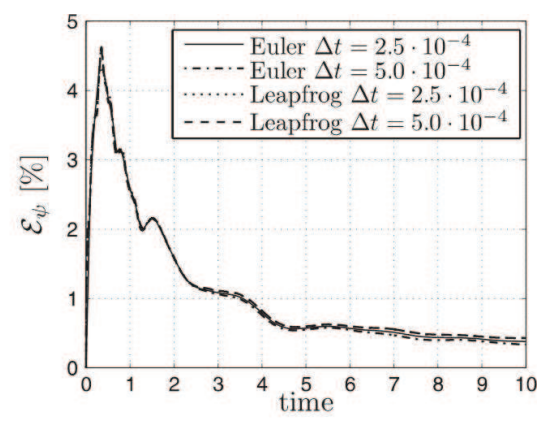

(b)

Figure 3: Two-dimensional linear system $\left(N_{p}=1641\right)$ : (a) influence of Courant number $C_{r}$ on the relative error $\mathcal{E}_{\psi}$ with respect to time and a forward Euler scheme, (b) the time evolution of the relative error $\mathcal{E}_{\psi}$ for two different integration schemes and two different time steps.

The accuracy and the convergence of the method have been also illustrated with two-dimensional nonlinear systems, as a Duffing-Van der Pol oscillator in [47].

\subsection{Probability of exceedance of a nonlinear SDOF oscillator}

The transient FPK equation finds a potential interest in seismic design. In this topic, reliability and probability of failure are usual questions. The estimation of a probability of exceedance is therefore required. The resolution of FPK equation with SPH method is applied to a structure damped by nonlinear fluid viscous devices and subjected to seismic loading. Fluid dampers installed on civil 
structures are commonly used as energy sinks for seismic protection. The nonlinear behavior of the damping device deeply influences the response of the structure [53].

The structure is supposed to mainly respond in its first mode and a nonlinear viscous damper is considered to mitigate the vibrations due to an earthquake. The equation of motion for the modal coordinate $q$ is

$$
\ddot{q}+2 \xi \omega_{0} \dot{q}+\eta \operatorname{sign}(\dot{q})|\dot{q}|^{\alpha}+\omega_{0}^{2} q=a W
$$

where $\omega_{0}$ and $\xi$ are the natural circular frequency and the damping ratio of the system, respectively. The nonlinear viscous force is characterized by the damper exponent $\alpha$ typically ranging from 0.2 to 1 [54] and $\eta$ the damping coefficient (related to the generalized mass of the system). The excitation is modeled by a white noise $W(t)$ modulated by a time window $a(t)$ defined as

$$
a(t)=\left\{\begin{array}{cc}
\left(\mathrm{t} / \mathrm{t}_{1}\right)^{n_{1}}, & t \leq t_{1} \\
1, & t_{1}<t<t_{2} \\
\exp \left(n_{2}\left(t_{2}-t\right)\right) & t_{2} \leq t
\end{array}\right.
$$

Thus, the only non-zero element of the diffusion matrix is $D_{22}(t)=S_{0} a^{2}$ with $E[W(t) W(t+\tau)]=$ $2 S_{0} \delta(\tau)$. The approximation of the probability of exceedance $P_{f}$ developed in Section 3.5 is illustrated in this example with an outcome space defined as $\Omega_{\mathcal{T}}=\{q \in \mathbb{R}:|q|>\mathcal{T}>0\}$ with $\mathbf{x}=[q \dot{q}]^{\mathrm{T}}$ the state vector of the system with $\mathcal{T}$ a threshold related to the ductility of the system.

The parameters have the numerical values: $\omega_{0}=\pi, \xi=1 \%, \eta=0.25, \alpha=0.35$ and $S_{0}=0.2$. The time window is characterized by $n_{1}=1, t_{1}=2, n_{2}=2$ and $t_{2}=6$. The initial uncorrelated Gaussian distribution has standard deviations equal to 0.15 for $q$ and 0.10 for $\dot{q}$ and mean values equal to zero. The number of particles is 1650 and the Courant number is 0.8 . Initially, particles are regularly arranged in a rectangle with edge sizes of 1.2 and 0.8 in $q$ and $\dot{q}$ directions, respectively.

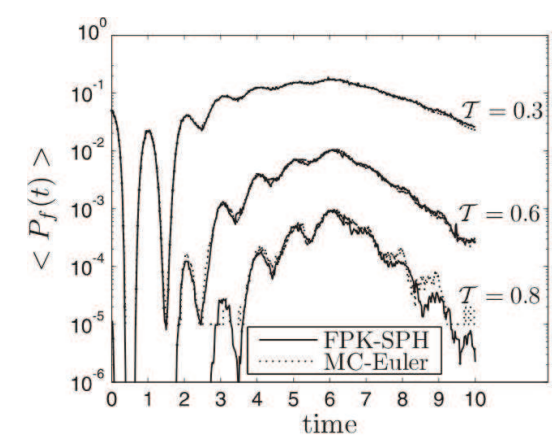

(a)

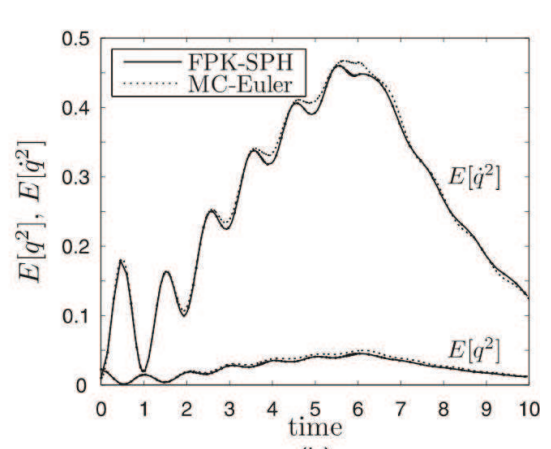

(b)

Figure 4: SDOF system with nonlinear viscous damper $\left(N_{p}=1650\right)$. The probability of exceedance $P_{f}$ (a) for the system (35) is estimated for different thresholds $\mathcal{T}$. The results obtained by solving FPK with SPH are compared with Monte-Carlo (MC) simulations ( $10^{5}$ samples). The estimated second-order moments of $q$ and $\dot{q}$ are compared with simulations (b).

Figure 4-a shows $\left\langle P_{f}\right\rangle$ for different thresholds $\mathcal{T}$ calculated with FPK equation and Equation (23). These results are compared with Monte-Carlo simulations ( $10^{5}$ samples and $2^{16}$ time steps). For the Mont-Carlo method, Equation (35) is integrated with a forward Euler scheme [6]. This figure shows the good agreement between results obtained with both methods, especially for high probabilities of exceedance. As expected, the convergence of stochastic simulations for low probabilities (in the order of $10^{-4}$ ) is not achieved. Because the response is not ergodic, a larger number of simulations is required to obtain accurate results. However, the computation of FPK equation has required about 11000 adaptive time steps and a single simulation has covered six orders of magnitude. Figure 4-b shows the estimation of the second-order moments of $q$ and $\dot{q}$ estimated with Equation (17) and highlights the 
ability of the method to capture the oscillations of the moments (and therefore in the system response). Some small differences with simulation results are observed, but the second-order moments of $q$ and $\dot{q}$ are properly estimated, despite an order of magnitude of difference between them, because the pdf is more stretched in the $\dot{q}$ direction than in the $q$ direction.

\subsection{Three-dimensional Lorenz Oscillator}

In this second example, the Lorenz attractor [55], a three-dimensional nonlinear oscillator, is studied. Governing equations are

$$
\begin{aligned}
\dot{x} & =\sigma(y-x)+W_{1} \\
\dot{y} & =\rho x-y-x z+W_{2} \\
\dot{z} & =x y-\beta z+W_{3}
\end{aligned}
$$

with $\sigma=10, \rho=28, \beta=8 / 3$ and $W_{1}, W_{2}, W_{3}$ three independent unit Gaussian $\delta$-correlated noises with $E\left[W_{i}(t) W_{j}(t+\tau)\right]=\delta_{i j} \delta(\tau)$. This example allows to present the method on a more complex system. Figure 5 illustrates the positions of the 9261 particles in the three-dimensional state space at four time steps. The use of different colors is a didactic way to represent the density associated with each particle. This example shows how the particles initially regularly spread out can produce complex distributions. Starting from a Gaussian initial distribution, particles move in the three-dimensional space to finally produce the steady-state distribution, i.e. the butterfly wings of the Lorenz attractor.

This example shows the adaptability of the method to follow a pdf with large distortions compared with the initial distribution and to manage the interaction between particles when their relative distance is reduced. The Eulerian kernel also shows its interest in the present application. The particles are initially distributed in a cube, then they are chaoticly divided into two groups. This fact highlights the capability of the method to deal with very low density zones and with distribution becoming discontinuous. In the steady state, the particles are moving on a surface and the interaction pattern has been significantly changed compared to the regular initial state. 


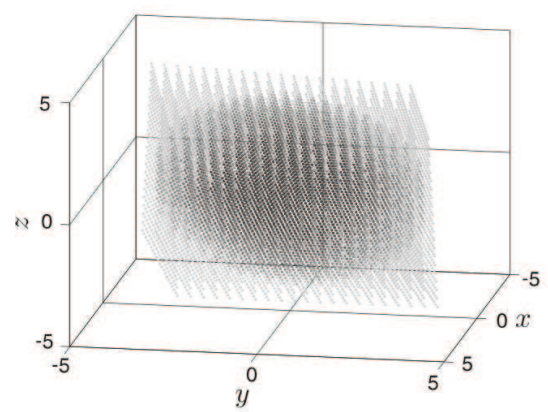

(a)

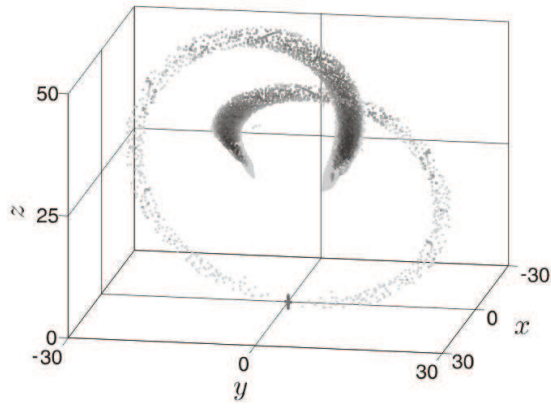

(c)

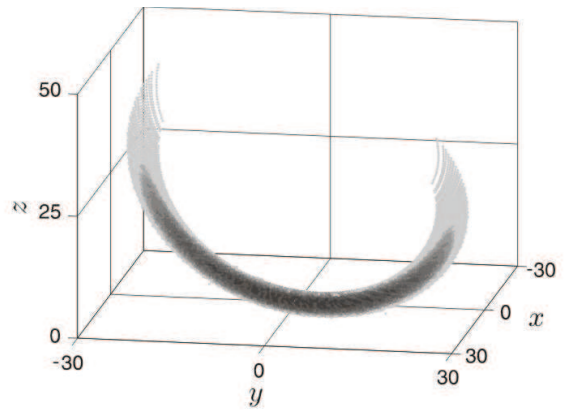

(b)

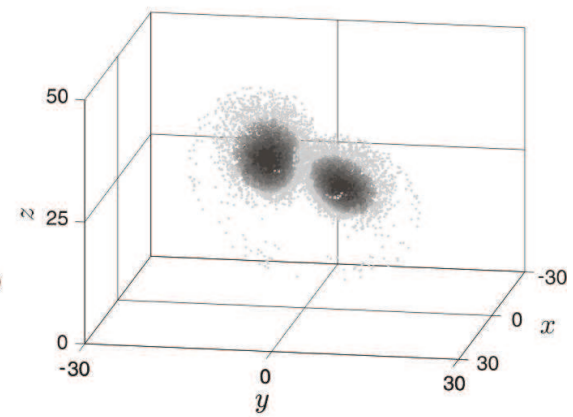

(d)

Figure 5: Probability density function $\psi$ of Lorenz oscillator calculated with 9261 particles at four different time steps: (a) $t=0.01$, (b) $t=0.2$, (c) $t=0.5$ and (d) $t=3.0$. The darker the larger pdf, the lighter the lower pdf.

It is now clear that moving particles provide an interesting way to represent the zones of the state space with higher density with a limited number of degrees of freedom whereas mesh-based methods would require to mesh a substantially larger volume. About the computation time, the CPU time for one iteration with 9261 particles is about one second (single processor $2.3 \mathrm{GHz}$ Intel i5); about 30000 steps have been performed in total for this simulation.

Figure 6 shows the time evolution of the second-order and sixth-order moments of the state variable $z$ calculated with Equation (18). The comparison with Monte-Carlo simulations highlights the validity of the rectangle approximation of moments for a reasonable number of particles. Of course, the calculation of the whole pdf provides a wider panel of information about the response of the random system, in particular about cross-moments.

The accurate representation of a high-order moment suggests an acceptable representation of the tails of the distribution with a limited number of particles. Also 30,000 samples are not sufficient for the Monte-Carlo simulation to converge in the estimation of the sixth-order moment. 


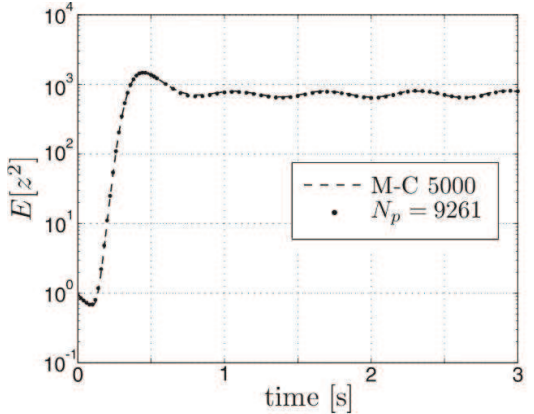

(a)

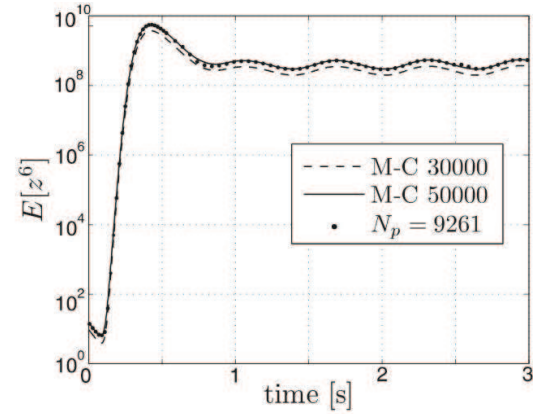

(b)

Figure 6: Time evolutions of the second-order (a) and sixth-order (b) moments of $z$ with SPH method. Comparison with Monte-Carlo simulation : 5000 samples (a), 30000 and 50000 samples (b).

\subsection{Probability of exceedance of a hysteretic oscillator}

As another application in earthquake engineering, the resolution of FPK equation with SPH method is applied to an SDOF system presenting a nonlinear elastoplastic behaviour and subjected to a transient seismic loading. This example aims at showing that the proposed method gives accurate information about the tail distribution through a reliability problem as in Section 4.2.

The equations of motion for a second-order structural system with hysteretic behavior [56] are

$$
\left\{\begin{array}{c}
\ddot{q}+2 \xi \omega_{0} \dot{q}+\Phi=a W \\
\Phi=\omega_{0}^{2}(\alpha q+(1-\alpha) z) \\
\dot{z}=\dot{q}\left(A-(\beta \operatorname{sign}(\dot{q} z)+\gamma)|z|^{n}\right)
\end{array}\right.
$$

with $q$ the dimensionless coordinate of the SDOF system, $\xi$ the structural damping ratio and $\omega_{0}$ the natural circular frequency. The excitation is a white noise $W(t)\left(E[W(t) W(t+\tau)]=2 S_{0} \delta(\tau)\right)$ modulated by a time window $a(t)$ and $\Phi(q, z)$ is the elastoplastic restoring force. This force is a convex combination of a linear stiffness component and a hysteretic component depending on the hardening parameter $\alpha$. The hysteretic behavior is controlled by the Bouc-Wen model [57] characterized by the dimensionless variable $z$ gathering the loading history and by the four parameters $A, \gamma, \beta$ and $n$ [56]. The outcome space is defined as $\Omega_{\mathcal{T}}=\{q \in \mathbb{R}:|q|>\mathcal{T}>0\}$ for different values for the threshold $\mathcal{T}$.

In this example inspired by $[1,58]$, the parameters of the system are chosen as $\omega_{0}=\pi, \xi=5 \%$, $\alpha=0.5$ and $S_{0}=1.0$ and those of the Bouc-Wen model are $A=1, \gamma=0.5, \beta=0.5, n=1$. This set of data provides a significantly nonlinear response. The time window is chosen as

$$
a(t)=f_{\max } \frac{t}{t_{\max }} \exp \left(1-\frac{t}{t_{\max }}\right)
$$

with $t_{\max }=2$ and $f_{\max }=1$. The initial uncorrelated Gaussian distribution is slightly dispersed with standard deviations equal to 0.05 and means equal to zero for $q, \dot{q}$ and $z$. The number of particles is $9261\left(=21^{3}\right)$ and the Courant number is 0.8. Initially, particles are regularly arranged in a cube with an edge size of 0.4 , and thus with 21 particles along each dimension. 


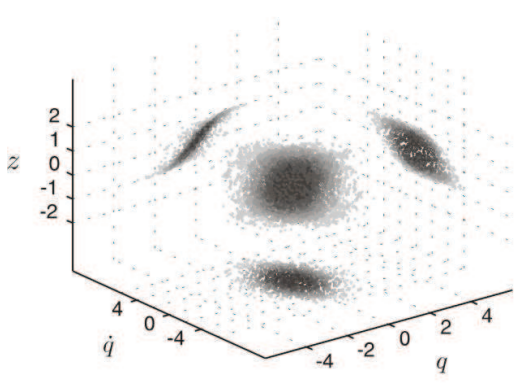

(a)

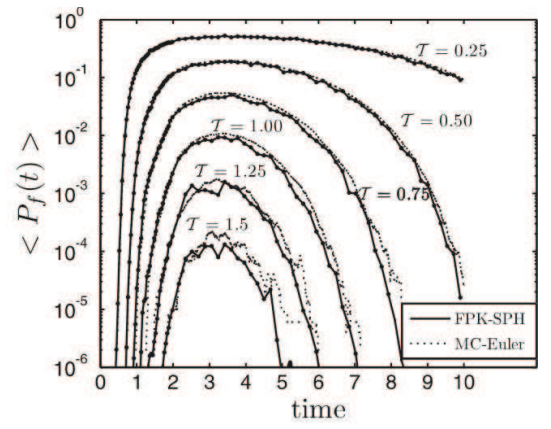

(b)

Figure 7: (a) Probability density function $\psi$ of the elastoplastic oscillator calculated with 9261 particles at time $t=3.6$ corresponding to the maximum variance. The darker the larger pdf, the lighter the lower pdf. Side and bottom views are projections on lateral faces; they illustrate the saturation effect on $z \in[-1 ; 1]$ although the solution space is $\mathbb{R}^{3}$. (b) Probability of exceedance $P_{f}$ for system (38) estimated for different thresholds $\mathcal{T}$. Results obtained with SPH are compared with Monte-Carlo (MC) simulations ( $5 \cdot 10^{5}$ samples).

This example shows in a three-dimensional state space that information about the tail distribution is accurately obtained with a reasonable number of particles during the whole duration of the earthquake, with a number of time steps limited to 35000. With only one run, the proposed method covers six orders of magnitude in the probability of exceedance, see Fig. 7-b, whilst the Monte Carlo simulation, presented here with $5 \cdot 10^{5}$ runs, poorly estimates probabilities lower than $10^{-5}$. It would require approximately $10^{8}$ runs to capture probabilities less than .

In the SPH simulation, the number of particles can be increased to smooth the mass distribution and consequently further ameliorate the estimation of low probabilities of exceedance. Also these results must be analyzed under the light of the significant dispersion from the slightly spread initial Gaussian distribution and the probability function illustrated in Figure 7-a; as an indication the second-order moments of $q$ and $\dot{q}$ are multiplied by 80 and 500, respectively, from the initial condition to their maximum values $(\mathrm{t}=3.6 \mathrm{~s})$. The more the probability is stretched compared with the initial distribution, the greater the number of particles is required. However, as illustrated in Figure 7-b, very good accuracy on the probability of exceedance for $\Omega_{\mathcal{T}}$ is achieved with few particles.

Besides this apparent robustness regarding the adaptation of the particle field from initial to transient states, the SPH algorithm proves to be efficient in containing the particles in the authorized subspace of the response domain. Indeed, the history variable $z$ in this model is known to be limited to $z \in[-A ; A]$. Although no specific boundary condition is imposed, i.e. particles are thus free the move in the state space according to the governing equations, the SPH algorithm contains particles in the subspace limited by planes $z= \pm 1$, as shown by the S-shaped projection of the pdf in the $(q, z)$ plane.

These examples illustrating well-known mechanical and civil engineering applications show that the resolution of the FPK equation with SPH may be seen as an interesting alternative to simulation methods.

\section{Conclusions}

In this paper, a contribution in the topic of the resolution of FPK equation with SPH method has been exposed. Some results in two and three dimensions are shown to illustrate the relevance of the proposed developments and assumptions. To honestly conclude, some advantages and limitations are summarized.

The Lagrangian formalism makes the method robust to deal with a large range of initial conditions even very different from the steady-state distribution. With SPH method, particles move from the initial to the stationary distribution by themselves. Therefore, only an accurate representation of the 
initial condition (even slightly dispersed) must be worried about. Furthermore, the implemented SPH method ensures the positivity of the pdf.

Nevertheless, the method has also some limitations. For instance, the stationary distribution cannot be directly computed (contrarily to finite element methods) which makes it more suited to transient problems. In the context of extreme value problems, the SPH method is limited because particles are not necessary located in low density zones. Nevertheless, illustrations have shown that high-order moments could be reasonably well estimated. From a computational point of view, for a large number of particles, the computation of the interaction can turn out to be time consuming and the recourse to advanced cell mapping or sorting algorithm could be mandatory.

Although being mainly exploratory, this work throws light on a new possible use of the SPH method. As presented in this paper, the method offers already the possibility to rapidly and easily extend the formalism to multidimensional spaces and to have an accurate representation of the transient regime of the FPK equation.

Finally, to the authors' knowledge, application of SPH technique to the FPK equation is the first opportunity to develop this method in a $n$-dimensional space, with no limitation on $n$ other than the capacity of the processors.

\section{Acknowledgment}

The authors would like to acknowledge the National Fund for Scientific Research of Belgium for its support. The first author would like to personally acknowledge Angelo Mariani for his kind attention and his precious advices. They will never be forgotten.

[1] Y.K. Lin and G.Q. Cai. Probabilistic Structural Dynamics. Advanced Theory and Applications. McGraw-Hill, New-York, 2nd edition, 2004.

[2] B. Oksendal. Stochastic differential equations. Springer-Verlag, 3rd edition, 1992.

[3] H. Risken. The Fokker-Planck Equation. Methods of solution and applications. Springer-Verlag, 2nd edition, 1996.

[4] R.L. Stratonovitch. Topics in the Theory of Random Noise, volume 1. Gordon and Breach Science Publishers, New-York, 1st edition, 1963.

[5] E. Wong and M. Zakai. On the relation between ordinary and stochastic differential equations. International Journal of Engineering Sciences, 3:213-229, 1965.

[6] D.P. Kroese, T. Taimre, and Z.I. Botev. Handbook of Monte Carlo Methods. Wiley series in probability and statistics, 2011.

[7] M. Grigoriu. Stochastic Calculus. Applications in Science and Engineering. Birkhauser, Boston, 1st edition, 2002.

[8] J.B. Roberts and P.D. Spanos. Random Vibration and Statistical Linearization. Dover Publications, New-York, dover edition edition, 2003.

[9] C. Proppe, H. J. Pradlwarter, and G. I. Schuëller. Equivalent linearization and Monte-Carlo simulation in stochastic dynamics. Probabilistic Engineering Mechanics, 18(1):1-15, 2003.

[10] C. Soize. Steady-state solution of Fokker-Planck equation in high dimension. Probabilistic Engineering Mechanics, 3(4):196-206, 1988.

[11] J. S. Chang and G. Cooper. A practical difference scheme for Fokker-Planck equations. Journal of Computational Physics, 6:1-16, 1970. 
[12] L. Pichler, A. Masud, and L. A. Bergman. Numerical solution of the Fokker-Planck equation by finite difference and finite element methods - a comparative study. In Proceeding of the Third Conference on Computational Methods in Structural Dynamics and Earthquake Engineering COMPDYN 2011, Corfu,Greece, May 2011.

[13] B.F. Spencer and L.A. Bergman. On the numerical solution of the Fokker-Planck equation for nonlinear stochastic systems. Nonlinear Dynamics, 4:357-372, 1993.

[14] R.S. Langley. A finite element method for thes tatistics of random nonlinear vibration. Journal of Sound and Vibration, 101:41-54, 1985.

[15] S.F. Wojtkiewicz and L.A. Bergman. Numerical solution of high dimensional Fokker-Planck equations. In 8th ASCE Specialty Conference on Probabilistic Mechanics and Structural Reliability, NotreDame, IN, USA, 2000.

[16] H.P. Langtangen. A general numerical solution method for Fokker-Planck equations with applications to structural reliability. Probabilistic Engineering Mechanics, 6:33-81, 1991.

[17] M. Kumar, P. Singla, J. Junkins, and S. Chakravorty. A multi-resolution meshless approach to steady state uncertainty determination in nonlinear dynamical systems. In Proceeding of the 38th IEEE Southeastern Symposium on Systems Theory, Cookeville, TN, USA, March 2006.

[18] M. Kumar, S. Chakravorty, P. Singla, and J. L. Junkins. The partition of unity finite element approach with hp-refinement for the stationary Fokker-Planck equation. Journal of Sound and Vibration, 327(1-2):144-162, 2009.

[19] M. Kumar, S. Chakravorty, and J. L. Junkins. A semianalytic meshless approach to the transient Fokker-Planck equation. Probabilistic Engineering Mechanics, 25(3):323-331, 2010.

[20] A. Lozinski and C. Chauviière. A fast solver for Fokker-Planck equation applied to viscoelastic flows calculations: 2d fene model. Journal of Computational Physics, 189(2):607-625, 2003.

[21] C. Chauvière and A. Lozinski. Simulation of dilute polymer solutions using a Fokker-Planck equation. Computers \& Fluids, 33(5-6):687-696, 2004.

[22] F. Chinesta, G. Chaidron, and A. Poitou. On the solution of Fokker-Planck equations in steady recirculating flows involving short fiber suspensions. Journal of Non-Newtonian Fluid Mechanics, 113(2-3):97-125, 2003.

[23] A. Ammar, B. Mokdad, F. Chinesta, and R. Keunings. A new family of solvers for some classes of multidimensional partial differential equations encountered in kinetic theory modeling of complex fluids. Journal of Non-Newtonian Fluid Mechanics, 139(3):153-176, 2006.

[24] A. Ammar, B. Mokdad, F. Chinesta, and R. Keunings. A new family of solvers for some classes of multidimensional partial differential equations encountered in kinetic theory modelling of complex fluids: Part II: Transient simulation using space-time separated representations. Journal of NonNewtonian Fluid Mechanics, 144(2-3):98-121, 2007.

[25] L. Chupin. Fokker-Planck equation in bounded domain. Annales de l'Institut Fourier, 60(1), 2010.

[26] A. Der Kiureghian. Structural reliability methods for seismic safety assessment: a review. Engineering Structures, 18(6):412-424, 1996.

[27] L. B. Lucy. A numerical approach to the testing of the fission hypothesis. Astronomical Journal, 82:1013-1024, 1977.

[28] R. A. Gingold and J.J. Monaghan. Smoothed particle hydrodynamics: theory and application to non-spherical stars. Monthly Notices of the Royal Astronomical Society, 181:375-389, 1977. 
[29] J. Feldman and J. Bonet. Dynamical refinement and boundary contact forces in SPH with applications in fluid flow problems. International Journal for Numerical Methods in Engineering, 72:295-324, 2007.

[30] J. P. Morris. Simulating surface tension with Smoothed particle hydrodynamics. International Journal for Numerical Methods in Fluids, 33(3):333-353, 2000.

[31] T. Rabczuk and J. Eibl. Simulation of high velocity concrete fragmentation using sph/mlsph. International Journal for Numerical Methods in Engineering, 56:1421-1444, 2003.

[32] T. Rabczuk and T. Belytschko. A three-dimensional large deformation meshfree method for arbitrary evolving cracks. Computer Methods in Applied Mechanics and Engineering, 196(2930):2777-2799, 2007.

[33] G. R. Johnson, R. A. Stryk, and S. R. Beissel. SPH for high velocity impact computations. Computer Methods in Applied Mechanics and Engineering, 139(1-4):347-373, 1996.

[34] P.W. Cleary and J.J. Monaghan. Conduction modelling using Smoothed particle hydrodynamics. Journal of Computational Physics, 148:227-264, 1999.

[35] J.H. Jeong, M.S. Jhon, J.S. Halow, and J. van Osdol. Smoothed particle hydrodynamics : Application to heat conduction. Computers Physics Communication, 153:71-84, 2003.

[36] P. W. Randles and L. D. Libersky. Smoothed particle hydrodynamics: Some recent improvements and applications. Computer Methods in Applied Mechanics and Engineering, 139:375-408, 1996.

[37] J. J. Monaghan. Smoothed particle hydrodynamics. Reports on Progress in Physics, 68:1703-1759, 2005.

[38] G.R. Liu and M.B. Liu. Smoothed Particle Hydrodynamics. A meshfree particle method. World Scientific Publ., 2003.

[39] M. Liu and G. Liu. Smoothed particle hydrodynamics (SPH): an overview and recent developments. Archives of Computational Methods in Engineering, 17(1):25-76, 2010.

[40] C.V. Chaubal, A. Srinivasan, O. Egecioglu, and L.G. van Leal. Smoothed particle hydrodynamics techniques for the solution of kinetic theory problems. Journal of Non-Newtonian Fluid Mechanics, 70:125-154, 1997.

[41] C. V. Chaubal and L. G. Leal. Smoothed particle hydrodynamics techniques for the solution of kinetic theory problems: Part 2. the effect of flow perturbations on the simple shear behavior of LCPs. Journal of Non-Newtonian Fluid Mechanics, 82(1):25-55, 1999.

[42] A. Ammar and F. Chinesta. A Particle Strategy for Solving the Fokker-Planck Equation Modelling the Fiber Orientation Distribution in Steady Recirculating Flows Involving Short Fiber Suspensions - Meshfree Methods for Partial Differential Equations II, volume 43 of Lecture Notes in Computational Science and Engineering, pages 1-15. Springer Berlin Heidelberg, 2005.

[43] E. Prulière. Modélisation de la microstructuration dans les polymères chargés. Application à la mise en forme. PhD thesis, Université Joseph Fourier, Grenoble, 2007.

[44] M.B. Liu, G.R. Liu, and K.Y. Lam. Constructing smoothing functions in smoothed particle hydrodynamics with applications. Journal of Computational and Applied Mathematics, 155:263$284,2003$.

[45] G. Lacombe. Analyse d'une équation de vitesse de diffusion. Comptes rendus de l'Académie des sciences, Math., 329:383-386, 1999. 
[46] P. Combis and J. Fronteau. A purely Lagrangian method for numerical integration of FokkerPlanck equation. Europhysics Letters, 2:227-232, 1986.

[47] T. Canor and V. Denoël. Transient Fokker-Planck equation solved with SPH. In Proceeding of the Fifth International Conference ACOMEN 2011, Liège, Belgium, November 2011.

[48] W.H. Press, S.A. Teukolsky, W.T. Vetterling, and B.P. Flannery. Numerical Recipes. The art of scientific computing. Cambridge University Press, 3rd edition, 2007.

[49] J. Swegle, J. Hicks, and S. Attaway. Smoothed particle hydrodynamics stability analysis. Journal of Computational Physics, 116:123-134, 1995.

[50] T. Belytschko, Y.. Guo, W. Kam Liu, and S. Ping Xiao. A unified stability analysis of meshless particle methods. International Journal for Numerical Methods in Engineering, 48(9):1359-1400, 2000.

[51] T. Rabczuk, T. Belytschko, and S. P. Xiao. Stable particle methods based on Lagrangian kernels. Computer Methods in Applied Mechanics and Engineering, 193(12-14):1035-1063, 2004.

[52] Y. Vidal, J. Bonet, and A. Huerta. Stabilized updated Lagrangian corrected SPH for explicit dynamic problems. International Journal for Numerical Methods in Engineering, 69(13):2687$2710,2007$.

[53] M. Di Paola, L. Mendola, and G. Navarra. Stochastic seismic analysis of structures with nonlinear viscous dampers. Journal of Structural Engineering, 133(10):1475-1478, 2007.

[54] G. Pekcan, J. B. Mander, and S. S. Chen. Fundamental considerations for the design of non-linear viscous dampers. Earthquake Engineering and Structural Dynamics, 28(11):1405-1425, 1999.

[55] H.K. Khalil. Nonlinear systems. Prentice Hall, 3rd edition, 2002.

[56] M. Ismail, F. Ikhouane, and J. Rodellar. The hysteresis Bouc-Wen model, a Survey. Archives of Computational Methods in Engineering, 16(2):161-188, 2009.

[57] Y.K. Wen. Method for random vibration of hysteretic systems. Journal of the Engineering Mechanics Division ASCE, 102(2):249-263, 1976.

[58] F. Ikhouane, V. Manosa, and J. Rodellar. Adaptive control of a hysteretic structural system. Automatica, 41(2):225-231, 2005. 